\title{
Curse or blessing? Influence of impurities on cross-coupling - Guideline for elucidating catalysts
}

\author{
Zoltán Novák ${ }^{1 *}$, Réka Adamik ${ }^{1}$, János T. Csenki ${ }^{1}$, Ferenc Béke ${ }^{1}$, Regina Gavaldik ${ }^{1}$, \\ Bálint Varga ${ }^{1}$, Bálint $\mathrm{Nagy}^{1}$, Zoltán May ${ }^{2 *}$, János Daru ${ }^{3 *}$, Zsombor Gonda ${ }^{4 *}$, Gergely L. Tolnai ${ }^{4 *}$ \\ ${ }^{1}$ ELTE "Lendület” Catalysis and Organic Synthesis Research Group, Institute of Chemistry, Eötvös \\ Loránd University, Pázmány Péter stny. 1/A, 1117 Budapest, Hungary, E-mail: novakz@elte.hu \\ web: http://zng.elte.hu/ \\ ${ }^{2}$ Research Centre for Natural Sciences, Eötvös Loránd Research Network. Magyar Tudósok körútja \\ 2, H-1117 Budapest, Hungary. E-mail: may.zoltan@ttk.hu \\ ${ }^{3}$ Lehrstuhl für Theoretische Chemie, Ruhr-Universität Bochum, 44780 Bochum, Germany. Email: \\ janos.daru@theochem.ruhr-uni-bochum.de \\ ${ }^{4}$ ELTE Tolnai Research Group, Institute of Chemistry, Eötvös Loránd University, Pázmány Péter \\ stny.1/A,1117 Budapest, Hungary,E-mail: gondazs@elte.hu, tolnai@chem.elte.hu \\ web: http://tolnai.chem.elte.hu
}

Several efforts have been made for the replacement of noble metal palladium in cross-coupling reactions, maintaining high efficiency of the target transformation. In several cases it is possible to perform the chemistry of palladium with related metals, and their activity was supported with mechanistic studies. Moreover, the complete exclusion of palladium is also in focus. Very recently it was demonstrated that special amine organocatalysts could catalyse Suzuki-Miyaura coupling reaction. Here we show that in this recent transformation homeopathic palladium impurities and trace phosphorous species originated from the conditions used for the organocatalyst synthesis are responsible for the catalytic effect instead of the amine species. This finding confirms the power of palladium in cross-coupling and draw the attention of impurity effect in this field of chemical research. In this article, we represent general guidelines for elucidating the real catalyst of reactions.

\section{Impurity effect on cross-coupling reactions}
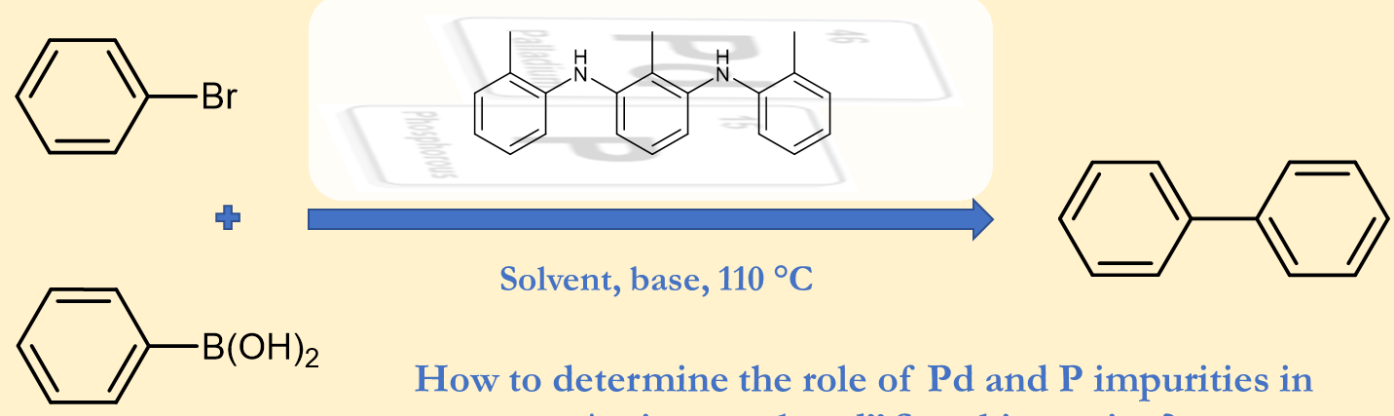

How to determine the role of $\mathbf{P d}$ and $\mathbf{P}$ impurities in „Amine-catalysed" Suzuki reaction? 
Cross-coupling reactions are one of the most powerful transformations in organic chemistry for the construction of new $\mathrm{C}-\mathrm{C}$ and $\mathrm{C}$-heteroatom bonds ${ }^{1,2}$ Among these transition metal catalysed reactions Suzuki-Miyaura coupling is probably the most useful ${ }^{3}$, moreover, this traditionally palladium catalysed coupling is the second most frequently used transformation in the field of medicinal chemistry ${ }^{4,5}$. Since the discovery of the coupling of $\mathrm{C}\left(\mathrm{sp}^{2}\right)$-halides and organic boron derivatives, tremendous efforts were made to the development of methodologies applicable on wide scope of substrates including API $\operatorname{targets}^{6,7}$. These developments focus on the modification and simplification of reaction parameters and the catalyst design to obtain highly active and selective catalysts for the transformation. Considering the latter aspects of methodology development, the replacement of palladium with other metals, the significant reduction of palladium loading down to "homeopathic" level or complete exclusion of any metals are the key areas of this research and development due to the relatively high cost of palladium, and sustainability reasons ${ }^{8}$. However, the realization of the alternative and improved methods is sometimes a really challenging task, and the greater the deviation from the original method, the more important it is to demonstrate experimental mechanistic proof of the new concept.

From mechanistic aspects the reduction of catalyst loading is the easiest to achieve, considering reproducibility and robustness. In this regard, there are many palladium catalysed methods which were efficiently performed with "homeopathic" $\mathrm{ppb}-\mathrm{ppm}$ level of palladium loading ${ }^{10}$, including the utilization of well characterized palladium complexes ${ }^{11-13}$ and nano-particles with different size ${ }^{14-17}$.

Depending on the reaction type, the replacement of a known transition metal catalyst with an alternative one is probably the most challenging task. In this situation, it should be proved that a metal can be replaced with another nonconventional one, defining its exact role in the catalytic cycle. It is well known historically that nickel ${ }^{18-24}$ and $\operatorname{copper}^{25-29}$ are able to act as a catalyst in cross-coupling reactions in a broad spectrum, therefore these metals are excellent, cheaper and earth abundant catalyst counterparts of palladium in organic syntheses.

The most important part of "catalyst replacement" research and development is the purity of the applied catalyst and additives participating in the reactions. In general, during the production of inorganic chemicals, different metals come hand-in-hand together, and simple metal salts with acceptable purity for organic chemistry $(>95 \%)$ are not appropriate for catalytic studies. For these, critical reaction components should be significantly purer $(>99.99 \%)$ to ensure the exclusion of possible contamination. Otherwise, the studies and results could lead to misinterpretation, driven by the illusion of discovery of a new catalytic phenomenon caused by trace amounts of impurities. A representative and edifying example of this effect is the attempted replacement of copper with iron in C-heteroatom bond forming coupling reactions ${ }^{30-32}$ Shortly after the disclosure of some "iron catalysed"-couplings Bolm and Buchwald revealed that the ppm level of copper impurities in iron are responsible of the key transformation, and ultra-pure iron salts did not catalyse the reaction at all (Fig. $1 \mathrm{a})^{33}$. The beneficial conclusion of these studies is that the couplings can be achieved with ppm level of copper catalysts, which is a significant development considering the catalyst loading demonstrated by Bolm and Norrby ${ }^{34}$.

Similarly, many developments of palladium-free Sonogashira coupling of terminal alkynes and aryl halides are precedented in the literature. The most frequently used alternative is copper ${ }^{35,36}$, but gold catalysed coupling was also developed ${ }^{37}$. However, later in both cases, $\mathrm{ppm} / \mathrm{ppb}$ level of palladium impurities ${ }^{38}$ were proved as the responsible catalyst by Echavarren and Espinet ${ }^{39}$, and by our research group (Fig. 1b) 
The impurities could be present in the catalyst, in the base or even in the solvent. Special care should be taken, especially in the labs where palladium is present in any form, to exclude ppb-ppm level crosscontamination. In our previous study ${ }^{40}$, we showed in the case of Sonogashira reaction, that the stir bar previously used in the Pd-catalysed reaction could transfer enough palladium into another reaction to reach complete conversion, even after cleaning (not with aqua regia). The stir bar problem as technical contamination source was deeply studied by Janiak ${ }^{41}$ and Ananikov ${ }^{42}$ and co-workers, and made solid statement on scientific basis on the role of metal impurities transported by this common laboratory accessory.

The complete omission of palladium catalyst from a traditional cross-coupling such as Suzuki reaction was also attempted ${ }^{43}$ earlier in 2003. However, shortly after the first examples of Pd-free Suzuki reaction, Leadbeater and co-workers reassessed their previous results (Fig. 1c), and clearly demonstrated that even ppb level of palladium is able to catalyse the Suzuki reaction of aryl halides and boronic acids under optimal reaction conditions ${ }^{44}$. These finding reverse the problem to benefit: a hyperactive catalytic system and useful method was discovered for the $\mathrm{C}-\mathrm{C}$ bond formation with the utilization of "homeopathic" amount of palladium catalyst.

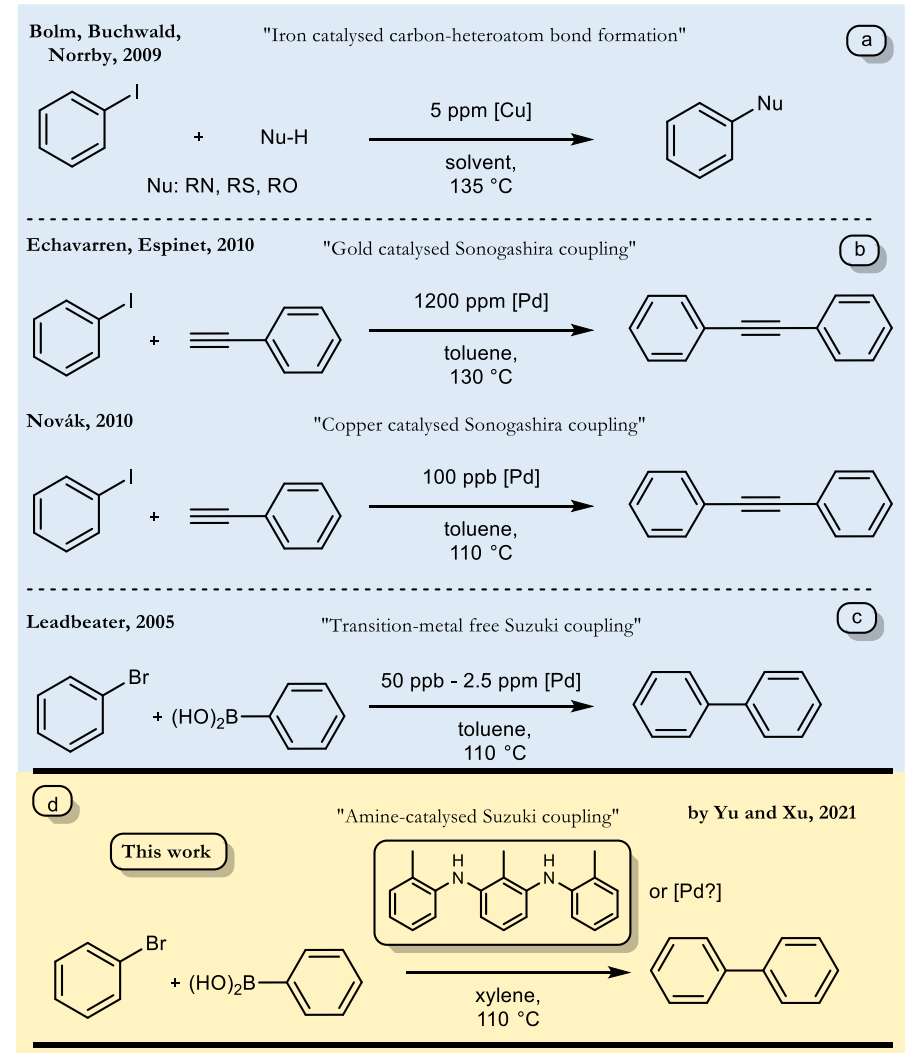

Fig. 1: Overview of "Replacement Catalysis" and "orgaNOmetallic" 45 couplings. a, Role of copper in "iron catalysed couplings. b, Role of Palladium in "gold" and "copper" catalysed" Sonogashira reactions. c, Role of palladium impurities in "metal-free" Suzuki-Miyaura coupling. d, Latest development of amine-catalysed organocatalytic Suzuki-Miyaura coupling.

Regarding the possible benefits, these kinds of catalytic developments are still in focus, hence researchers are experiencing same problems, challenges and difficulties. Very recently, $\mathrm{Yu}, \mathrm{Xu}$ and coworkers developed an amine-catalysed Suzuki-Miyaura-type coupling reaction (Fig. 1d) ${ }^{45}$. In their work, it was demonstrated that specially functionalized aniline derivatives could catalyse the reaction of aryl 
halides and aryl boronic acids to form biaryl scaffolds. According to their extensive research the traditional palladium catalysts for Suzuki-Miyaura reaction can be replaced with an organocatalytic alternative.

Considering the previous findings on the role of palladium impurities and the result of this novel organocatalytic procedure, we aimed to analyse and explore this amine-catalysed approach of biaryl construction on the basis of our earlier experience in "homeopathic" catalysis.

\section{Guideline, materials and methods}

To evaluate any hypothesis on the organocatalytic manner of a reaction it is utmost importance to be extremely careful in practical details regarding the utilized methods and materials. As a starting point of our research, we set up a general strategy and guideline for deep analysis with the highlight of critical points, potential risks of methodology developments (Fig. 2). Mechanistically, the most important issue is the identification of any possible metal source in the reaction which could provide potential catalysts into the system. Use of this guideline is even more recommended when reaction temperature exceeds $110^{\circ} \mathrm{C}$. According to our proposed guideline, the following general requirements should be considered to exclude unwanted contaminants from the reaction mixtures:

I. Potential risk: impurity of materials. The substrates and the bases can be originally contaminated or polluted with transition metals in a laboratory over a long period of time.

Recommendations:

1. The catalyst: it is forbidden to use the same metal for the synthesis of the organocatalyst, which metal is a known active catalyst of the transformation in focus.

2. Alternative metal catalyst, base and additives: both should have ultra-high purity $(>99.99 \%)$.

3. Substrates: the substrates should have the highest available purity, and should have reliable batch history and handling in the laboratory over the years;

4. Solvent: should be distilled.

II. Potential risk: metal enrichment and deposition on the equipment.

Highest potential risk: the stir bar. For mechanistic studies always use brand new PTFA coated stir bar.

Recommendations for the use of equipment such as vials, syringes, spatula, stir bar:

1. Use brand new and/or appropriately cleaned equipment.

2. Run blank reaction without any catalyst, to get zero reference and eliminate any background reactions. 


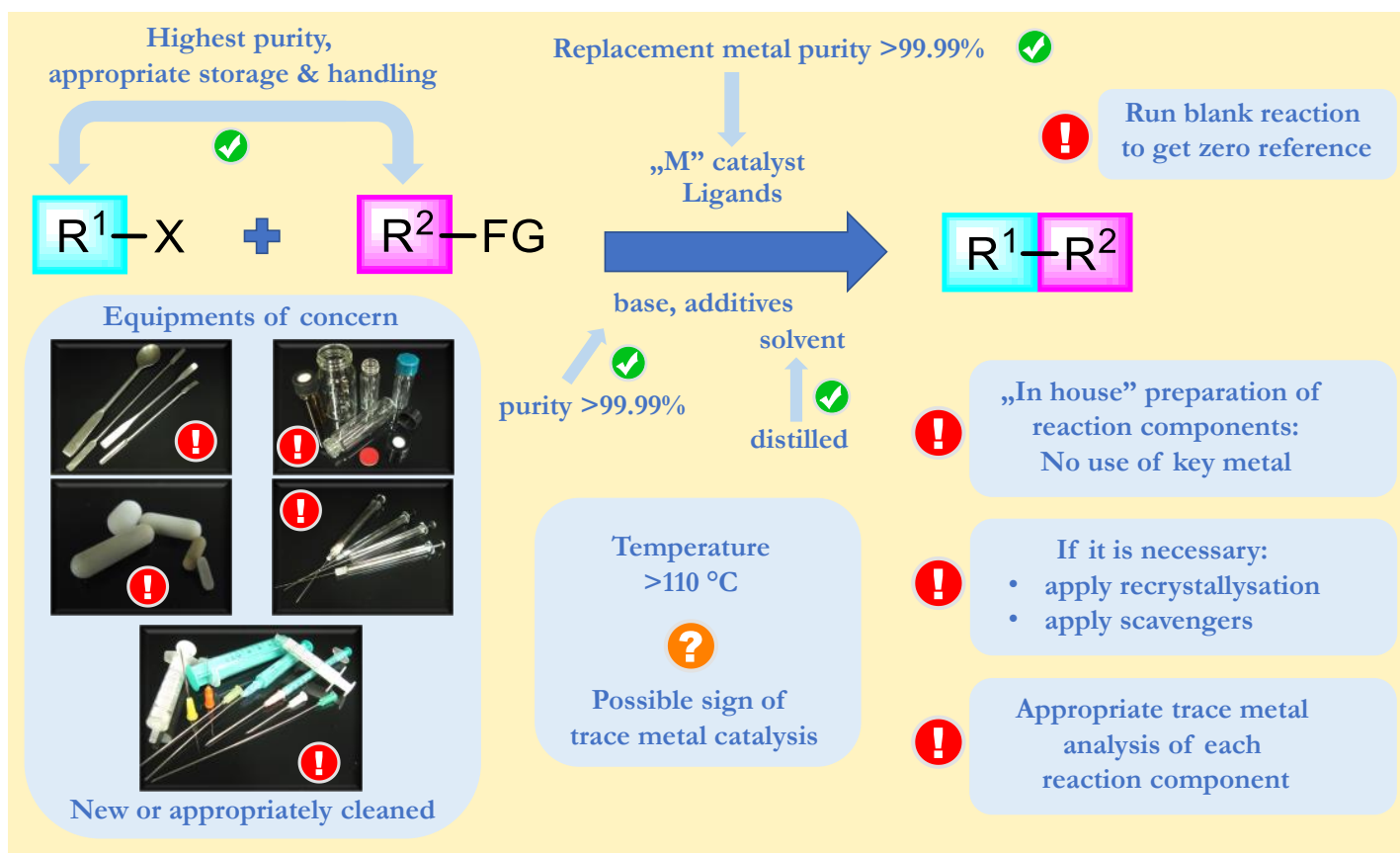

Fig. 2: Guideline for the future catalytic developments

\section{Results and discussions}

\section{Identification of critical issues with the aid of the proposed guideline}

According to our general guideline, the experimental detail of the recently developed amine-catalysed version of Suzuki reaction ${ }^{45}$ was evaluated. As a result of the procedure analysis, we found that the quality of used materials, the substrates and base were appropriately chosen for the research (Guideline I.2-4). Some reactions of the optimisation studies which were carried out in the presence of different amines resulted $0 \%$ conversion, which supports this ascertainment. We tried to identify any possible equipment issues (Guideline II.), but there is no appropriate information regarding the used equipment for the literature procedure, especially the treatment of stir bars during the project development.

As a result of our evaluation, we identified the synthesis and purity analysis of the amine catalyst as the most critical issues of this organocatalytic approach. The key compounds of the transformation were prepared in one or two sequential palladium catalysed reaction with a relatively high Pd-catalyst loading (3-5 mol\%), which violated I.1 bullet point of our guideline. It is well known in practice that products of transition metal catalysed reactions contain significant amount of metal impurities even after chromatographic purification ${ }^{6}$. To meet the purity requirements of the pharmaceutical sector these products should be treated, purified appropriately to remove Pd metal traces from the organic products $(<1 \mathrm{mg} / \mathrm{kg})$ especially from APIs ${ }^{48-49}$.

The determination of the palladium content of the amine products is a key point of the research. According to the current literature study ${ }^{45}$, the amine contained only sub $\mathrm{ppb}(<\mu \mathrm{g} / \mathrm{kg})$ level of Pd after chromatographic purification on the basis of the ICP-MS analysis results. However, closer look into the analytical method seemingly inappropriate conditions were used for the sample preparation including acidic digestion of the organic material and dissolution of Pd present, which caused the unreasonably low Pd content of either the amine or the examined reaction mixture. Thus, the Pd content determination method should be re-evaluated. 


\section{Experimental studies}

Focussing on the most critical point of the guideline and mechanistic considerations, beside the reproduction (reference procedure used $3 \mathrm{~mol} \% \mathrm{Pd}$ ) we aimed to prepare the most reactive aminecatalyst in a Buchwald-Hartwig reaction performed with lower palladium loading ( $0.1 \mathrm{~mol} \% \mathrm{Pd})$, and through an independent palladium-free way to obtain organic product relatively lower Pd content and surely without metal contaminant (Fig. 3a).

First, following the procedure of $\mathrm{Yu}$ and $\mathrm{Xu}$ we prepared the most efficient diamine catalyst through Buchwald-Hartwig coupling of 2,6-diaminotoluene (1) and 2-bromotoluene (2) in the presence of 3 $\mathrm{mol} \%$ of $\mathrm{Pd}$ and tricyclohexylphosphine ligand without difficulties (AmineCat-30). As an extension of the literature procedure, we prepared the target amine catalyst performing an additional recrystallisation of the Buchwald-Hartwig product obtained after column chromatographic purification (AmineCat-30-RC).

Then, we had to change the reaction conditions to perform the same amination reaction with significantly lower palladium loading, because the conditions of the original protocol did not allow the use of palladium catalyst lower than $3 \mathrm{~mol} \%$ amount to obtain the target amine with acceptable efficiency. To solve the Pd loading problem of the original approach we developed an aqueous-micellar procedure for the coupling of 2,6-diaminotoluene and 2-bromotoluene using Kolliphor ${ }^{50}$ as a surfactant additive. With the aid of this reaction condition, we successfully achieved the coupling in the presence of $1000 \mathrm{ppm}$ palladium catalyst and isolated the amine product (AmineCat-1) in 91\% yield after the chromatographic purification. Then, one batch of the product was further purified by recrystallisation to obtain purer amine product (AmineCat-1-RC) for analysis and reactivity study. It is worth noting that the appearance of the amine products was changed spectacularly after recrystallisation (Fig. 3a).

Finally, we aimed to prepare the aniline derivative without the utilization of palladium. Based on our experience in the field of hypervalent iodonium chemistry ${ }^{51-53}$ we designed a transition metal-free protocol $^{54}$ for the construction of the key amine starting form 2,6-diaminotoluene and di-otolyliodonium triflate. However, the $\mathrm{N}$-arylation took place very slowly, and after 24 hours reaction time the desired amine was obtained only in $7 \%$ yield, but provided a perfect metal free sample (AmineCat-0) for further studies.

After having five different samples of the same amine, we measured their trace metal content with ICP-OES and -MS analysis (Fig. 3b). 20-50 mg of each sample (3 parallels were measured) was treated with the mixture of 6-6 mL of concentrated $\mathrm{HNO}_{3}$ (Suprapur, 65\%) and $\mathrm{HCl}$ (Suprapur, 30\%) at 200 ${ }^{\circ} \mathrm{C}$ (20 bar in autoclave) for $2 \mathrm{~h}$. The trace metal analysis revealed that the amine sample (AmineCat30) made via the literature procedure contains significantly higher Pd content compared to the results of literature analysis $(519 \mathrm{mg} / \mathrm{kg}$ vs. $<1 \mu \mathrm{g} / \mathrm{kg})$. Additionally, our ICP-OES analysis revealed that this sample contained $136 \mathrm{mg} / \mathrm{kg}$ phosphorous. This is a breakthrough discovery for the interpretation of the activity of the key amine. The palladium and the phosphorous together are required for the amination, thus various Pd-P species as ligated palladium catalyst can elute with the amine through the chromatographic purification of the crude product. Consequently, the amine is contaminated by both metal and phosphorous species, and could act as possible catalysts for the Suzuki-Miyaura reaction. Simple recrystallisation of this amine product (AmineCat-30) obtained by the literature procedure resulted white crystalline substance (AmineCat-30-RC) which contained $11.3 \mathrm{mg} / \mathrm{kg}$ Pd by ICP-MS and phosphorous below the detection limit of ICP-OES $(<20 \mathrm{mg} / \mathrm{kg})$. This result demonstrates the efficiency of recrystallisation to show that additional purification step is required to remove $\mathrm{Pd}-\mathrm{P}$ 
complexes from amines. Coupling under the micellar conditions in the presence of $1000 \mathrm{ppm} \mathrm{Pd}$ loading ensured lower Pd and P content of the product AmineCat-1 $(353 \mathrm{mg} / \mathrm{kg}$ Pd and $101 \mathrm{mg} / \mathrm{kg}$ P respectively), especially after recrystallisation (AmineCat-1-RC has $<0.16 \mathrm{mg} / \mathrm{kg}$ Pd and $<20 \mathrm{mg} / \mathrm{kg}$ P content). The Pd-free method gave amine sample AmineCat-0 with even lower Pd and P content $(0.08 \mathrm{mg} / \mathrm{kg} \mathrm{Pd}$ and $<20 \mathrm{mg} / \mathrm{kg} \mathrm{P}$ ).

In parallel, we used an efficient fluorometric detection method developed by Welch ${ }^{55}$ for the qualitative visualization of palladium impurities in AmineCats (Fig. 3d). This simple analytical method offers helpful, easy and fast visualisation way for organic chemists to detect palladium impurities in the samples even in $\mathrm{mg} / \mathrm{kg}$ level, therefore its use is recommended for related future studies. In our study, the green fluorescent colour clearly showed the presence of Pd in AmineCat-30, AmineCat-1, and AmineCat-30-RC (Fig. 3d).

We tested the reactivity of the prepared and analysed amines in the coupling of phenylboronic acid and 4-bromobenzonitrile under the optimized literature reaction conditions taking into account our guidelines. We found perfect reproducibility of the amine catalysed reaction with AmineCat-30 and we observed $98 \%$ conversion after 2 hours at $110{ }^{\circ} \mathrm{C}$ in 0 -xylene. The coupling reaction with AmineCat-1 under the same reaction conditions resulted slightly lower conversion (93\%). On the basis of this observation, we concluded that the Pd loading of the Buchwald-Hartwig coupling in the region of $0.1-3 \mathrm{~mol} \%$ used for the preparation of amine catalyst did not determine significantly the activity of the amine catalyst. Dramatic change was found in reactivity when the recrystallised amines (AmineCat-30-RC and AmineCat-1-RC) were used in the coupling reactions. Experiments provided $0 \%$ conversion of the desired transformation when these purified amines were used as catalysts, which is the first sign of the exclusion of organocatalytic reaction path. The same inactivity was observed in the reaction performed with AmineCat-0 which was prepared via non-Pd catalysed path.

The time-conversion curves were also determined for each reaction performed with different amine catalysts (Fig. 3d). This gave similar reaction profile for both AmineCat-30 and AmineCat-1, and the transformation is complete in 20 minutes. It worth noting that these two samples supply 70 and 50 ppm palladium into the reaction mixture respectively, calculated on the basis of the ICP-MS analysis results. In contrast, other amine catalysts with significantly lower Pd and P content showed no activity in these experiments as well, providing similar results to a blank reaction performed without amine.

The catalytic activity of each catalyst was tested in different solvents such as toluene, $t$-amylalcohol, $t$ amylalcohol/water mixture and in water, and we found similar activity trend among the catalysts, and concluded that the non-polar solvents (toluene and o-xylene) are preferential for the successful transformation. We observed reaction in polar protic solvents only in case of AmineCat-30 and AmineCat-1 having the highest palladium content ${ }^{56}$.

Next, we studied the sensitivity of the Suzuki-Miyaura coupling to palladium loading under the utilized reaction conditions with the controlled addition of well-defined Pd complex (Fig. 3e). 1-100 ppm $\mathrm{PdCl}_{2}\left(\mathrm{PPh}_{3}\right)_{2}$ was added to the reaction mixture and the conversions were determined after 2 hours. We found that the reaction was almost complete when the Pd loading was at least $25 \mathrm{ppm}$, and we observed no significant conversions change when $5 \mathrm{~mol} \%$ recrystallised amine AmineCat-30-RC or AmineCat-1-RC was present in the reaction mixture beside the well-defined added Pd-complex (Fig. $3 e)$. Consequently, the amine does not contribute to the catalytic performance of Pd. The timeconversion curves of these reactions (Fig. 3f) show good correlation between the reactivity and the palladium content of the reaction mixtures regardless the source of the palladium. 


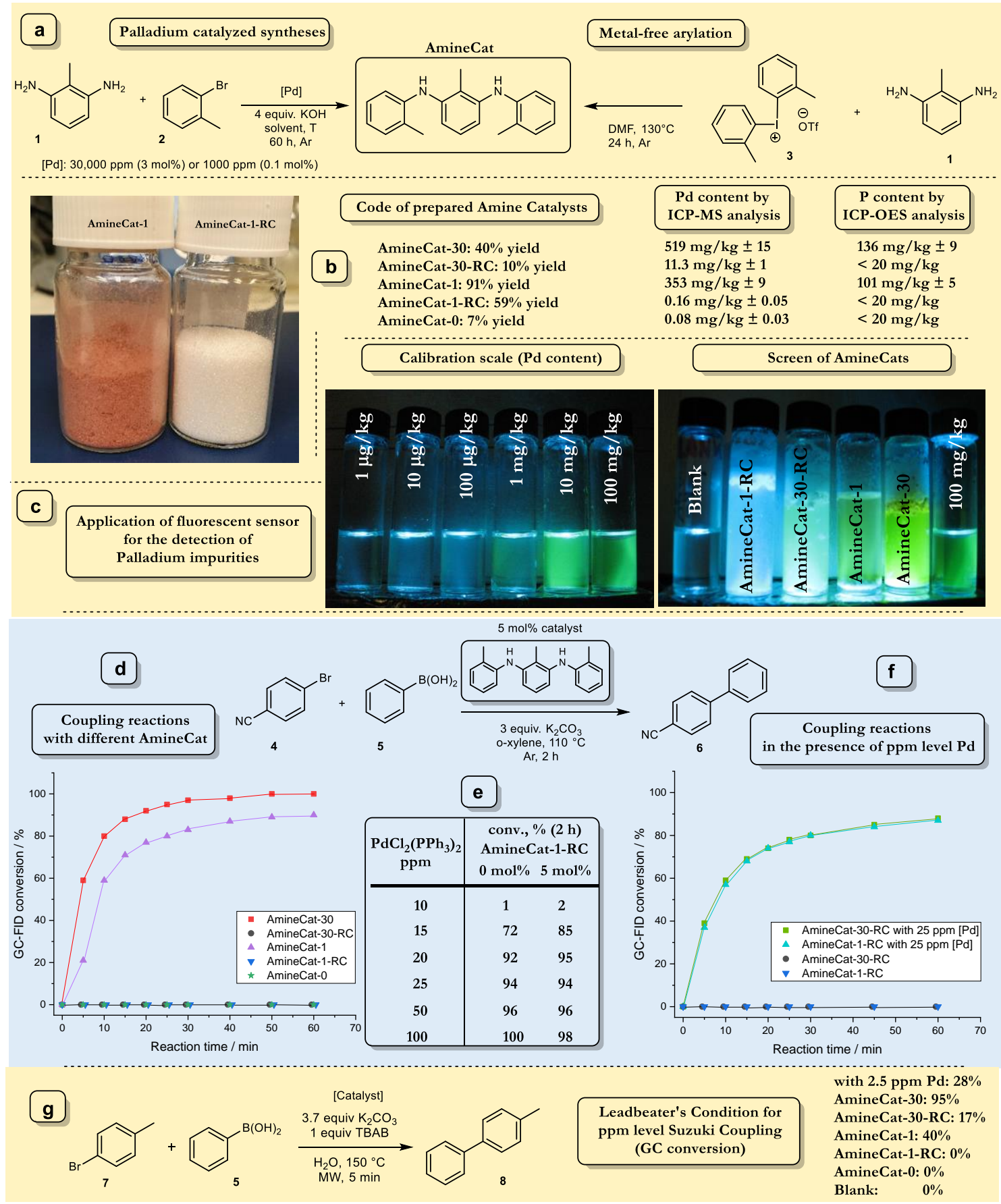

Fig. 3: Mechanistic studies of Amine-catalysed Suzuki-Miyaura coupling. a, Synthesis of different amin catalysts. AmineCat-30: Reproduction of literature procedure with 30,000 ppm (3 mol\%) Pd loading (amine was purified by column chromatography); AmineCat-30-RC: Reproduction of literature procedure with 30,000 ppm (3mol\%) Pd loading with additional ReCrystallisation of amine product obtained after column chromatography; AmineCat-1: Coupling with 1,000 ppm (0.1 mol\%) Pd loading, (amine was purified by column chromatography); AmineCat-1-RC: Coupling with 1,000 ppm $(0.1 \mathrm{~mol} \%)$ Pd loading with additional ReCrystallisation product obtained after column chromatography; AmineCat-0: Metal-free arylation with diaryliodonium salts. $\mathbf{b}$, Pd-content of the amines were determined by ICP measurements. $\mathbf{c}$, Pd content visualization with fluorescent probe. $\mathbf{d}$, Reaction monitoring with different amine catalysts. e, Reactions in the presence of controlled amount of $\mathrm{PdCl}_{2}\left(\mathrm{PPh}_{3}\right)_{2}$ with or without AmineCat-1-RC. $\mathbf{f}$, Reaction monitoring $\mathrm{PdCl}_{2}\left(\mathrm{PPh}_{3}\right)_{2}$ spiked AmineCats g, Leadbeater's condition for Suzuki coupling with ppm level of Pd. 
Comparison of Fig. 3d and Fig. 3f depict similar kinetics of the reactions, thus corroborates the preference of Pd-P catalysis over the amine catalysed transformation, and supports the high sensitivity of the reaction to Pd impurities.

Finally, we switched the reaction conditions to Leadbeater's approach, to study the activity of the amines in a completely different reaction media (Fig. 3g). In water, using microwave irradiation at 150 ${ }^{\circ} \mathrm{C}$ after 5 minutes the conversion of the reaction was $28 \%$ in the presence of $2.5 \mathrm{ppm} \mathrm{PdCl}_{2}\left(\mathrm{PPh}_{3}\right)_{2}$, while AmineCat-30, AmineCat-30-RC and AmineCat-1 gave 95\%, 17\% and 40\% conversion respectively. We observed no reaction with AmineCat-1-RC and AmineCat-0 similarly to the blank reference.

As further evidence of the Pd-contamination effect we tested randomly selected coupling partners (aryl bromides and boronic derivatives) and attempted their coupling with our AmineCats (Fig. 4.). We observed the same reactivity profile in each case of substrates. AmineCat-30 catalysed the SuzukiMiyaura coupling due to their ability to supply appropriate amount of palladium for the coupling, while reactions in the presence of AmineCats with lower Pd content did not take place at all.

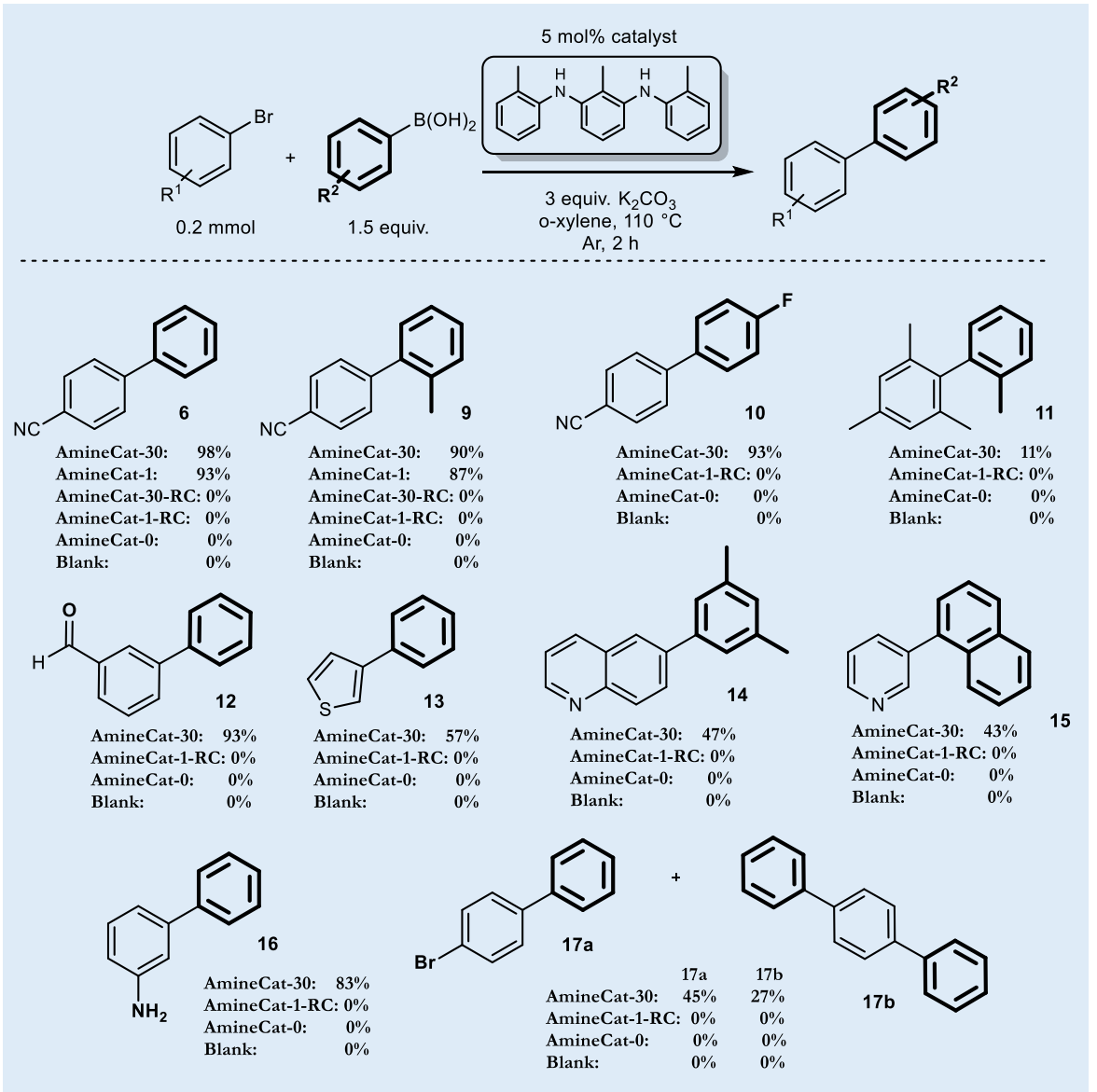

Fig. 4: Catalyst test with different coupling partners. Reaction conditions: AmineCat (3.0 mg, $0.01 \mathrm{mmol})$, bromobenzene derivative $(0.2 \mathrm{mmol})$, arylboronic acid $(0.3 \mathrm{mmol}), \mathrm{K}_{2} \mathrm{CO}_{3}(82.9 \mathrm{mg}, 0.6 \mathrm{mmol}) 110{ }^{\circ} \mathrm{C}$ for 2 hours. 


\section{Theoretical considerations}

It remains to understand why density functional theory (DFT) calculations are indicating the organocatalyzed reaction pathway to be plausible at $110{ }^{\circ} \mathrm{C}$, by estimating the apparent activation barrier to be $28.5 \mathrm{kcal} / \mathrm{mol}$, in spite of the diminishing conversion in absence of palladium as demonstrated above. To this end we would like to emphasize that although there is no standard procedure to directly model a solid additive via molecular quantum chemical calculations, omitting the estimation of the solvation free energy $\left(\Delta \Delta \mathrm{G}_{\text {sol }}\right)$ for $\mathrm{K}_{2} \mathrm{CO}_{3}$ in toluene with respect to the bulk solid reference, and thus describing it as a genuine solution-phase reactant results in an apparently activated species with overestimated interactions ${ }^{57}$. One commonly applied strategy is to calculate $\Delta \Delta G_{\text {sol }}$ via a thermodynamic cycle, combining both experimental and calculated data, however this approach features large changes in the electronic structure, therefore prone to the inherent errors of DFT ${ }^{57}$. Here we follow an alternative approach ${ }^{58}$ by providing a lower bound to the $\Delta \Delta G_{\text {sol }}$ through modelling the changed interactions by the dissociation of a $\mathrm{K}_{2} \mathrm{CO}_{3}$ cluster model $\left(1 / \mathrm{n}\left(\mathrm{K}_{2} \mathrm{CO}_{3}\right)_{\mathrm{n}} \rightarrow \mathrm{K}_{2} \mathrm{CO}_{3}\right)$. In order to obtain compatible results with $\mathrm{Yu}, \mathrm{Xu}$ et $a l_{.}^{45}$ we have re-evaluated these dissociation free energies with the exact same functional and basis set ${ }^{56}$, yielding $19.5 \mathrm{kcal} / \mathrm{mol}$ for $\mathrm{n}=4$. By including this lower bound estimate of $\Delta \Delta \mathrm{G}_{\mathrm{sol}}$ to the mechanism of $\mathrm{Yu}, \mathrm{Xu}$ et al. we already obtain a prohibitively high $37.9 \mathrm{kcal} / \mathrm{mol}$ activation barrier for the organocatalytic process (see Fig. 1 in SI). Note, that based on the transition state theory the reaction is expected to be on the order of $2 * 10^{5}$ times slower due to the additional $9.4 \mathrm{kcal} / \mathrm{mol}$ apparent barrier height, which is sufficient to rationalise the experimental behaviour even considering the approximate nature of our $\Delta \Delta \mathrm{G}_{\mathrm{sol}}$ estimate. Note that the insolubility of $\mathrm{K}_{2} \mathrm{CO}_{3}$ in refluxing xylene has been experimentally confirmed in our laboratory ${ }^{56}$.

\section{Conclusions}

In conclusion, following our guideline we demonstrated that the recently developed "amine-catalysed" Suzuki-Miyaura coupling reaction in focus took place with the participation of $\mathrm{Pd}$ and $\mathrm{P}$ impurities supplied by the amine component, ruling out the role of amine as organocatalyst in the transformation. As an additional information the experimental results clearly show that even phosphorous derivatives are present in the organic products even after chromatographic purification step, supposedly due to the co-elution of various Pd-phosphine species with the Buchwald-Hartwig products. We composed a guideline as a recommendation for similar future methodology developments to highlight the importance of appropriate handling of materials and equipment, purification and analysis of products in order to avoid any mechanistic misinterpretations. Consideration of the critical issues could save time and energy for future studies started on a promising way paved by misleading background. We hope this approach could turn the curse of any impurity effect to the blessing of a novel and highly efficient catalyst systems developed in chemical research, which is the case of the present observation. The recently developed methodology emphasizes the greatness of Suzuki-Miyaura reaction which provides access to versatile and complex molecules including API-s with a "homeopathic" amount of palladium loading and the selection of appropriate reaction conditions, even on large scale. 


\section{Materials and Methods}

All experiments were carried out with new or specially treated glassware, stirrer bars, spatula, septa. For details see Supporting information

\section{Procedure for the synthesis of AmineCat-30 and AmineCat-30-RC (Condition A)}

2-methylbenzene-1,3-diamine (1.22 g, $10.0 \mathrm{mmol}, 1.0$ equiv), $\mathrm{PdCl}_{2}\left(\mathrm{PCy}_{3}\right)_{2}(220 \mathrm{mg}, 0.3 \mathrm{mmol}, 0.03$ equiv) and $\mathrm{KOH}$ (2.24 g, 40 mmol, 4.0 equiv) were placed in a reaction flask. The flask was sealed, then evacuated and backfilled with argon three times. Degassed toluene was added with a syringe $(50 \mathrm{~mL})$ followed by 1-bromo-2-methylbenzene $(4.8 \mathrm{~mL}, 6.84 \mathrm{~g}, 40 \mathrm{mmol}, 4.0$ equiv). The reaction mixture was placed into a pre-heated oil bath and stirred at 110 ${ }^{\circ} \mathrm{C}$ for 60 hours. The reaction mixture was cooled to room temperature, filtered through a celite pad and diluted with acetone. The mixture was concentrated to silica and the product was isolated with flash column chromatography on $\mathrm{SiO}_{2}$, using hexane and ethyl acetate as eluents, to give AmineCat-30 (1209 mg, 4 mmol, 40\% yield) as a brownish solid.

AmineCat-30-RC was obtained from another batch after recrystallisation from ethyl acetate/ hexane (1:4) (302 mg, 1 mmol, $10 \%$ yield) as a white solid.

\section{Procedure for the synthesis of AmineCat-1 and AmineCat-1-RC (Condition B)}

2-methylbenzene-1,3-diamine (1.22 g, 10.0 mmol, 1.0 equiv.), bis[cinnamyl palladium(II)] chloride $(5.2 \mathrm{mg}, 0.01$ mmol) tBuXPhos $(17 \mathrm{mg}, 0.04 \mathrm{mmol})$ and $\mathrm{KOH}(2.24 \mathrm{~g}$, 40 mmol, 4.0 equiv) were placed in a reaction flask. The flask was sealed, then evacuated and back-filled with argon three times. Degassed Kolliphor EL-solution (2 wt\% in water) was added with a syringe ( $50 \mathrm{~mL}$ ) followed by 1-bromo-2-methylbenzene (4.8 mL, 6.84 g, $40 \mathrm{mmol}, 4.0$ equiv.). The reaction mixture was placed into a pre-heated oil bath and stirred at $50^{\circ} \mathrm{C}$ for 60 bours. The reaction mixture was cooled to room temperature, extracted with $(3 \times 50 \mathrm{~mL})$, the organic phase was washed with brine and dried on $\mathrm{Na}_{2} \mathrm{SO}_{4}$. The crude product was purified by flash column chromatography on $\mathrm{SiO}_{2}$ using hexane and ethyl acetate as eluents, to give AmineCat-1 (2757 mg, 9.1 mmol, 91\% yield), as a mauve solid.

AmineCat-1-RC was obtained from another batch after recrystallisation from ethyl acetate/ bexane (1:4) (1799 mg, 5.9 mmol, $59 \%$ yield) as a white solid.

\section{Procedure for the synthesis of AmineCat-O (Conditions C)}

Under inert atmosphere, 2-methylbenzene-1,3-diamine $(549.8 \mathrm{mg}, 4.5 \mathrm{mmol})$ was added to di-o-tolyliodonium tetrafluoroborate $(3.563 \mathrm{~g}, 9 \mathrm{mmol})$ in anh. DMF $(45 \mathrm{~mL})$ and the resulting mixture heated at $130^{\circ} \mathrm{C}$ for $24 \mathrm{~b}$ in a pre-heated oil bath. After cooling, water $(50 \mathrm{~mL})$ was added and the mixture extracted with diethyl-ether $(3 \times 50 \mathrm{~mL})$. The organic extracts were combined and washed with more water $(200 \mathrm{~mL})$, dried on $\mathrm{MgSO}_{4}$ and the volatiles were removed in vacuo to give the crude product as a brown oil. The crude product was purified by flash column chromatography on $\mathrm{SiO}_{2}$ using hexane and ethyl acetate as eluents, to give AmineCat-0 as crystalline solid (91.3 mg, $0.30 \mathrm{mmol}, 7 \%$ ).

\section{General procedure for the coupling reactions}

The respective Amine Cat (3 mg, $0.01 \mathrm{mmol}$ ), bromobenzene derivative (0.2 mmol) if solid, arylboronic acid (0.3 mmol), $\mathrm{K}_{2} \mathrm{CO}_{3}(83 \mathrm{mg}$, $0.6 \mathrm{mmol}$ ) / Alfa Aesar, Puratronic (99.997\%)/ were placed in a septum-capped reaction vial. The vial was sealed, then evacuated and back-filled with argon three times. Degassed o-xylene was added with a syringe (3.0 $m L)$ and finally bromobenzene derivative $(0.2 \mathrm{mmol})$ if liquid. The reaction mixture was placed into a pre-heated aluminium block and stirred at $110{ }^{\circ} \mathrm{C}$ for the indicated time. 


\section{Author contributions}

G. L. T., Z. N., Z. M., J. D. and F. B. took part in the composition of manuscript. G. L. T., Z. N. and Zs. G. directed the research. R. A., F. B., J. T. Cs., R. G., B. V., Zs. G. performed the experiments. Z. M. performed the ICP analysis. J. D. performed the DFT studies. All authors participated in the final composition of the manuscript.

\section{Competing Interests}

The authors declare no competing interests.

\section{Acknowledgements}

The Authors thank the support of National Research, Development and Innovation Office (Grant No. K132077, K124592, K130048). G. L. T. is grateful for Janos Bolyai Research Scholarship of the Hungarian Academy of Sciences (BO/536/20) and ÚNKP-20-5. This work was completed in the ELTE Institutional Excellence Program supported by the National Research, Development and Innovation Office (NKFIH-1157-8/2019-DT). The Authors thank. Mr. Kristof Hegedüs at Research Center for Natural Sciences, Budapest, for the assistance of NMR measurements, and for Zsuzsanna Czégény (Research Center for Natural Sciences, Budapest) for the read-through of the manuscript.

\section{Data availability}

The authors declare that the main data supporting the findings of this study are available within the article and its Supplementary Information file. Extra data are available from the corresponding author upon request.

\section{References}

1. de Meijere, A. \& Diederich, F. Metal-Catalyzed Cross-Coupling Reactions. vol. 1 (WILEY-VCH Verlag GmbH \& Co. KGaA, Weinheim, 2004).

2. Negishi, E. Handbook of Organopalladium Chemistry for Organic Synthesis. (John Wiley \& Sons, Inc., 2002).

3. Miyaura, N. \& Suzuki, A. Palladium-catalyzed cross-coupling reactions of organoboron compounds. Chem. Rev. 95, 2457-2483 (1995).

4. Wu, X.-F., Anbarasan, P., Neumann, H. \& Beller, M. From noble metal to Nobel Prize: palladiumcatalyzed coupling reactions as key methods in organic synthesis. Angew. Chem. Int. Ed. 49, 90479050 (2010).

5. Brown, D. G. \& Boström, J. Analysis of Past and Present Synthetic Methodologies on Medicinal Chemistry: Where Have All the New Reactions Gone? J. Med. Chem. 59, 4443-4458 (2015). 
6. Magano, J. \& Dunetz, J. R. Large-Scale Applications of Transition Metal-Catalyzed Couplings for the Synthesis of Pharmaceuticals. Chem. Rev. 111, 2177-2250 (2011).

7. Blakemore, D. Chapter 1. Suzuki-Miyaura Coupling. in Synthetic Methods in Drug Discovery 1-69 (Royal Society of Chemistry, 2015).

8. Hudson, R. \& Katz, J. L. Toward the Selection of Sustainable Catalysts for Suzuki-Miyaura Coupling: A Gate-to-Gate Analysis. ACS Sustain. Chem. Eng. 6, 14880-14887 (2018).

9. de Vries, A. H. M., Mulders, J. M. C. A., Mommers, J. H. M., Henderickx, H. J. W. \& de Vries, J. G. Homeopathic Ligand-Free Palladium as a Catalyst in the Heck Reaction. A Comparison with a Palladacycle Org. Lett. 5, 3285-3288 (2003).

10. Alimardanov, A., de Vondervoort, L. S.-v., de Vries, A. H. M. \& de Vries, J. G. Use of "Homeopathic" Ligand-Free Palladium as Catalyst for Aryl-Aryl Coupling Reactions. Adv. Synth. Catal. 346, 1812-1817 (2004).

11. Bedford, R. B., Hazelwood (née Welch), S. L., Horton, P. N. \& Hursthouse, M. B. Orthopalladated phosphinite complexes as high-activity catalysts for the Suzuki reaction. Dalton Trans. 4164-4174 (2003).

12. Beller, M., Fischer, H., Herrmann, W. A., Öfele, K. \& Brossmer, C. Palladacycles as Efficient Catalysts for Aryl Coupling Reactions. Angew. Chem. Int. Ed. 34, 1848-1849 (1995).

13. Tang, Y., Zeng, Y., Hu, Q., Huang, F., Jin, L., Mo, W., Sun, N., Hu, B., Shen, Z., Hu, X. \& Sun, W.-H. Efficient Catalyst for Both Suzuki and Heck Cross-Coupling Reactions: Synthesis and Catalytic Behaviour of Geometry- Constrained Iminopyridylpalladium Chlorides. Adv. Synth. Catal. 358, 2642-2651 (2016).

14. Eremin, D. B. \& Ananikov, V. P. Understanding active species in catalytic transformations: From molecular catalysis to nanoparticles, leaching, "Cocktails" of catalysts and dynamic systems. Coord. Chem. Rev. 346, 2-19 (2017).

15. Deraedt, C. \& Astruc, D. "Homeopathic" Palladium Nanoparticle Catalysis of Cross CarbonCarbon Coupling Reactions. Acc. Chem. Res. 47, 494-503 (2013).

16. Kunfi, A., May, Z., Németh, P. \& London, G. Polydopamine supported palladium nanoparticles: Highly efficient catalysts in Suzuki cross-coupling and tandem Suzuki cross-coupling/nitroarene reductions under green reaction conditions. J. Catal. 361, 84-93 (2018). 
17. Enneiymy, M., Drian, C. L., Ghimbeu, C. M. \& Becht, J.-M. Mesoporous carbon supported ultrasmall palladium particles as highly active catalyst for Suzuki-Miyaura reaction. Appl. Organomet. Chem. (2019)

18. Clevenger, A. L., Stolley, R. M., Aderibigbe, J. \& Louie, J. Trends in the Usage of Bidentate Phosphines as Ligands in Nickel Catalysis. Chem. Rev. 120, 6124-6196 (2020).

19. Buchspies, J. \& Szostak, M. Recent Advances in Acyl Suzuki Cross-Coupling. Catalysts 9, 53 (2019).

20. Dander, J. E. \& Garg, N. K. Breaking Amides using Nickel Catalysis. ACS Catal. 7, 1413-1423 (2017).

21. Maluenda, I. \& Navarro, O. Recent Developments in the Suzuki-Miyaura Reaction: 2010-2014. Molecules 20, 7528-7557 (2015).

22. Han, F.-S. Transition-metal-catalyzed Suzuki-Miyaura cross-coupling reactions: a remarkable advance from palladium to nickel catalysts. Chem. Soc. Rev. 42, 5270-5298 (2013).

23. Rosen, B. M., Quasdorf, K. W., Wilson, D. A., Zhang, N., Resmerita, A.-M., Garg, N. K. \& Percec, V. Nickel-Catalyzed Cross-Couplings Involving Carbon-Oxygen Bonds. Chem. Rev. 111, 13461416 (2011).

24. Baqi, Y. Recent Advances in Microwave-Assisted Copper-Catalyzed Cross-Coupling Reactions. Catalysts 11, 46 (2020).

25. Ma, D. \& Cai, Q. Copper/Amino Acid Catalyzed Cross-Couplings of Aryl and Vinyl Halides with Nucleophiles. Acc. Chem. Res. 41, 1450-1460 (2008).

26. Beletskaya, I. P. \& Cheprakov, A. V. Copper in cross-coupling reactions. Coord. Chem. Rev. 248, $2337-2364$ (2004).

27. Qiao, J. \& Lam, P. Copper-Promoted Carbon-Heteroatom Bond Cross-Coupling with Boronic Acids and Derivatives. Synthesis 2011, 829-856 (2010).

28. Beletskaya, I. P. \& Cheprakov, A. V. The Complementary Competitors: Palladium and Copper in C-N Cross-Coupling Reactions. Organometallics 31, 7753-7808 (2012).

29. Evano, G., Blanchard, N. \& Toumi, M. Copper-Mediated Coupling Reactions and Their Applications in Natural Products and Designed Biomolecules Synthesis. Chem. Rev. 108, 3054 3131 (2008). 
30. Correa, A., Carril, M. \& Bolm, C. Iron-Catalyzed S-Arylation of Thiols with Aryl Iodides. Angew. Chem. Int. Ed. 47, 2880-2883 (2008).

31. Bistri, O., Correa, A. \& Bolm, C. Iron-Catalyzed C-O Cross-Couplings of Phenols with Aryl Iodides. Angew. Chem. Int. Ed. 47, 586-588 (2008).

32. Correa, A. \& Bolm, C. Iron-Catalyzed N-Arylation of Nitrogen Nucleophiles. Angew. Chem. Int. Ed. 46, 8862-8865 (2007).

33. Buchwald, S. L. \& Bolm, C. On the Role of Metal Contaminants in Catalyses with FeCl3. Angew. Chem. - Int. Ed. 48, 5586-5587 (2009).

34. Larsson, P.-F., Correa, A., Carril, M., Norrby, P.-O. \& Bolm, C. Copper-Catalyzed CrossCouplings with Part-per-Million Catalyst Loadings. Angew. Chem. - Int. Ed. 48, 5691-5693 (2009).

35. Murashkina, A. V., Mitrofanov, A. Y. \& Beletskaya, I. P. Copper in Cross-Coupling Reactions: I. Sonogashira-Hagihara Reaction. Russ. J. Org. Chem. 55, 1445-1458 (2019).

36. Kanwal, I., Mujahid, A., Rasool, N., Rizwan, K., Malik, A., Ahmad, G., Shah, S. A. A., Rashid, U. \& Nasir, N. M. Palladium and Copper Catalyzed Sonogashira cross Coupling an Excellent Methodology for C-C Bond Formation over 17 Years: A Review. Catalysts 10, 443 (2020).

37. González-Arellano, C., Abad, A., Corma, A., García, H., Iglesias, M. \& Sánchez, F. Catalysis by Gold(I) and Gold(III): A Parallelism between Homo- and Heterogeneous Catalysts for CopperFree Sonogashira Cross-Coupling Reactions. Angew. Chem. - Int. Ed. 46, 1536-1538 (2007).

38. Plenio, H. Catalysts for the Sonogashira Coupling-The Crownless Again Shall Be King. Angew. Chem. - Int. Ed. 47, 6954-6956 (2008).

39. Lauterbach, T., Livendahl, M., Rosellon, A., Espinet, P. \& Echavarren, A. M. Unlikeliness of PdFree Gold(I)-Catalyzed Sonogashira Coupling Reactions. Org. Lett. 12, 3006-3009 (2010).

40. Gonda, Z., Tolnai, G. L. \& Novák, Z. Dramatic Impact of ppb Levels of Palladium on the “Copper-Catalyzed” Sonogashira Coupling. Chem. Eur. J. 16, 11822-11826 (2010).

41. Vollmer, C., Schröder, M., Thomann, Y., Thomann, R. \& Janiak, C. Turning Teflon-coated magnetic stirring bars to catalyst systems with metal nanoparticle trace deposits - A caveat and a chance. Appl. Catal., A 425-426, 178-183 (2012). 
42. Pentsak, E. O., Eremin, D. B., Gordeev, E. G. \& Ananikov, V. P. Phantom Reactivity in Organic and Catalytic Reactions as a Consequence of Microscale Destruction and ContaminationTrapping Effects of Magnetic Stir Bars. ACS Catal. 9, 3070-3081 (2019).

43. Leadbeater, N. E. \& Marco, M. Transition-Metal-Free Suzuki-Type Coupling Reactions: Scope and Limitations of the Methodology. J. Org. Chem. 68, 5660-5667 (2003).

44. Arvela, R. K., Leadbeater, N. E., Sangi, M. S., Williams, V. A., Granados, P. \& Singer, R. D. A Reassessment of the Transition-Metal Free Suzuki-Type Coupling Methodology. J. Org. Chem. 70, 161-168 (2005).

45. Leadbeater, N. E. When is free really free? Nat Chem 2, 1007-1009 (2010).

46. Xu, L., Liu, F.-Y., Zhang, Q., Chang, W.-J., Liu, Z.-L., Lv, Y., Yu, H.-Z., Xu, J., Dai, J.-J. \& Xu, H.-J. The amine-catalysed Suzuki-Miyaura-type coupling of aryl halides and arylboronic acids. Nat. Catal. 4, 71-78 (2021).

47. Garrett, C. E. \& Prasad, K. The Art of Meeting Palladium Specifications in Active Pharmaceutical Ingredients Produced by Pd-Catalyzed Reactions. Adv. Synth. Catal. 346, 889-900 (2004).

48. Phillips, S., Holdsworth, D., Kauppinen, P. \& Namara, C. M. Palladium Impurity Removal from Active Pharmaceutical Ingredient Process Streams. Johnson Matthey Technol. Rev. 60, 277-286 (2016).

49. It is important to note that, in this current amine catalysed transformation the post treatment of amine catalysts after their preparation with thiol-scavenger did not affected the efficiency of the key Suzuki-type coupling according to the comparative reactivity study.

50. Mattiello, S., Rooney, M., Sanzone, A., Brazzo, P., Sassi, M. \& Beverina, L. Suzuki-Miyaura Micellar Cross-Coupling in Water, at Room Temperature, and under Aerobic Atmosphere. Org. Lett. 19, 654-657 (2017).

51. Novák, Z., Aradi, K., Tóth, B. \& Tolnai, G. Diaryliodonium Salts in Organic Syntheses: A Useful Compound Class for Novel Arylation Strategies. Synlett 27, 1456-1485 (2016).

52. Olofsson, B., Ilan, M. \& Zvi, R. The Chemistry of Hypervalent Halogen Compounds. Patai's Chemistry of Functional Groups (Wiley-Blackwell, 2019).

53. Merritt, E. \& Olofsson, B. Diaryliodonium salts: a journey from obscurity to fame. Angew. Chem. Int. Ed. 48, 9052-9070 (2009). 
54. Carroll, M. A. \& Wood, R. A. Arylation of anilines: formation of diarylamines using diaryliodonium salts. Tetrahedron 63, 11349-11354 (2007).

55. Bu, X., Koide, K., Carder, E. J. \& Welch, C. J. Rapid Analysis of Residual Palladium in Pharmaceutical Development Using a Catalysis-Based Fluorometric Method. Org. Process Res. Dev. 17, 108-113 (2012).

56. See details in the Supplementary information

57. Yaman, T. \& Harvey, J. N. Suzuki-Miyaura coupling revisited: an integrated computational study. Faraday Discuss. 220, 425-442 (2019).

58. Li, B.-W., Wang, M.-Y., Fang, S. \& Liu, J.-Y. DFT Study on the Mechanism of Palladium(0)Catalyzed Reaction of Aryl Iodides, Norbornene, and Di-tert-butyldiaziridinone Organometallics 38, 2189-2198 (2019). 